\title{
Successful synchronous chemotherapy and radiotherapy followed by consecutive chemotherapy without surgery for primary intracranial choriocarcinoma: A case report
}

\author{
MINJIN KIM*, JINA YUN*, SOON MI HUR, JONG JOO MOON, JAE HYUNG NAM, SE HYUNG KIM, \\ HYUN JUNG KIM, CHAN KYU KIM, SEONG KYU PARK and DAE SIK HONG
}

Division of Hematology-Oncology, Department of Internal Medicine, Soonchunhyang University School of Medicine, Bucheon University, Bucheon, Gyeonggi 420-767, Republic of Korea

Received June 1, 2012; Accepted August 29, 2012

DOI: $10.3892 / \mathrm{ol} .2012 .901$

\begin{abstract}
We experienced a rare case of primary intracranial choriocarcinoma treated successfully with synchronous chemotherapy and radiotherapy followed by three consecutive courses of chemotherapy without surgery. A 19-year-old male patient presented with a two-week history of diplopia, headache, nausea and vomiting. Neurological examination revealed visual disturbance, bilateral hearing loss, bilateral sixth nerve palsy, left skew deviation, downgaze limitation and clockwise torsional nystagmus on the left upgaze. During image evaluation, the patient suddenly presented with a confused mental status, bradycardia and decreased respiration. An emergency third ventriculostomy was conducted, followed by synchronous ifosfamide, cisplatin and etoposide chemotherapy (ICE; I, $900 \mathrm{mg} / \mathrm{m}^{2}$; C, $20 \mathrm{mg} / \mathrm{m}^{2}$; and E, $60 \mathrm{mg} / \mathrm{m}^{2}$ ) on days $1-5$, and external radiotherapy (whole brain, $30 \mathrm{~Gy} / 15 \mathrm{Fxs}$; local boost, 30 Gy/15 Fxs) followed again by three consecutive courses of chemotherapy. This therapy resulted in tumor regression by $63 \%$ and full improvement in the patient's neurological condition. However, it was difficult to remove the remaining tumor using a surgical approach due to the risk of postoperative hemorrhage and limited access. We planned to observe the remaining mass without surgery. This patient is now alive and the mass size has not changed for 18 months since treatment. This is the first report of the effectiveness of synchronous chemotherapy and radiotherapy followed by
\end{abstract}

Correspondence to: Dr Dae Sik Hong, Division of HematologyOncology, Department of Internal Medicine, Soonchunhyang University School of Medicine, Bucheon University 1174 Jung-Dong, Wonmi-Gu, Bucheon, Gyeonggi 420-767, Republic of Korea

E-mail: 19983233@schmc.ac.kr

* Contributed equally

Key words: synchronous chemotherapy and radiotherapy, consecutive chemotherapies, primary intracranial choriocarcinoma consecutive chemotherapy without surgery in a patient with primary intracranial choriocarcinoma.

\section{Introduction}

Primary intracranial germ cell tumors (GCTs) are associated with many unanswered questions due to their low incidence (approximately $1.8-3.0 \%$ of all primary brain tumors) (1). They include germinomas (65\%), teratomas (18\%), embryonal carcinomas (5\%), endodermal sinus tumors $(7 \%)$ and choriocarcinomas (5\%) (2). While germinomas may be cured by chemotherapy and/or radiotherapy, non-germinatous malignant GCTs are highly resistant to standard treatments, and their prognosis is extremely poor. Of these, primary intracranial choriocarcinomas (PICCCs) are extremely rare. They have the worst prognosis and high early mortality rates, particularly due to tumor hemorrhage within the first year $(2,3)$. The clinical and biological behavior of PICCCs remains to be fully elucidated and, to date, no therapeutic strategy has been established. We successfully treated a case of PICCC with synchronous chemotherapy and radiotherapy followed by three consecutive chemotherapy courses without surgery. The study was approved by the ethics committee of Soonchunhyang University Bucheon hospital, Bucheon, Korea. Written informed patient consent was obtained from the patient.

\section{Case report}

A 19-year-old male patient presented with a two-week history of diplopia, headache, nausea and vomiting. Neurological examination revealed visual disturbance, bilateral hearing loss, bilateral sixth nerve palsy, left skew deviation, downgaze limitation and clockwise torsional nystagmus on the left upgaze. Computed tomography (CT) and magnetic resonance imaging (MRI) scans revealed acute hydrocephalus and a mass (38x30 mm) with foci of hemorrhage and calcification in the pineal region (Fig. 1A). Whole spine MRI and cerebrospinal fluid (CSF) cytology results demonstrated normal extraneural/CSF metastasis. The pituitary function survey revealed normal levels of growth hormone $(\mathrm{GH})$, follicle-stimulating 
hormone (FSH), luteinizing hormone (LH), vasopressin AVP) and prolactin (PRL). The patient suddenly presented with a confused mental status, bradycardia and decreased respiration, and underwent emergency third ventriculostomy to relieve some of the symptoms, which were caused by hydrocephalus blocking the flow of the spinal fluid in the brain. The baseline levels of serum $\beta$ human chorionic gonadotropin $(\beta-\mathrm{HCG})$ and $\alpha$-fetoprotein (AFP) were $10147 \mathrm{mIU} / \mathrm{ml}$ and $2.6 \mathrm{ng} / \mathrm{ml}$, respectively. The CSF $\beta-\mathrm{HCG}$ and AFP were $634 \mathrm{mIU} / \mathrm{ml}$ and $0.6 \mathrm{ng} / \mathrm{ml}$, respectively. Positron emission tomography-CT (PET-CT) enhanced abdominopelvic CT and chest CT were conducted, and no abnormal lesions were observed, with the exception of the pineal region. The patient underwent synchronous ifosfamide, cisplatin and etoposide chemotherapy (ICE; $\mathrm{I}, 900 \mathrm{mg} / \mathrm{m}^{2} ; \mathrm{C}, 20 \mathrm{mg} / \mathrm{m}^{2}$; and $\left.\mathrm{E}, 60 \mathrm{mg} / \mathrm{m}^{2}\right)$ on days $1-5$, and external radiotherapy (whole brain, $30 \mathrm{~Gy} / 15 \mathrm{Fxs}$; local boost, $30 \mathrm{~Gy} / 15 \mathrm{Fxs}$ ) followed by ventriculoperitoneal CSF shunting. Following completion of the first course of synchronous chemotherapy and radiotherapy, the serum $\beta$-HCG and AFP were normalized at $0.2 \mathrm{mIU} / \mathrm{ml}$ and $2.2 \mathrm{ng} / \mathrm{ml}$, respectively (Fig. 2). Imaging studies (CT and MRI) demonstrated an $\sim 45 \%$ reduction in the tumor mass $(21 \times 16 \mathrm{~mm})$ and disappearance of the hydrocephalus (Fig. 1B). Consequently, the patient underwent an additional three courses of chemotherapy with the same ICE regimen. The tumor mass was further reduced by $63 \%(14 \times 12 \mathrm{~mm}$; Fig. 1C), and the patient's abnormal neurological symptoms recovered fully. It was difficult to remove the remaining tumor using a surgical approach due to the risk of postoperative hemorrhage and limited access. We aimed to observe the remaining mass without surgery, and since then the patient has undergone regular CT/MRI follow-up as well as serum $\beta$-HCG and AFP level examinations every three months. The patient is now alive, the mass size has not changed for 18 months since treatment, and he is leading a normal life.

\section{Discussion}

PICCCs are extremely rare and represent only $0.05 \%$ of all brain tumors in Japan (4). A statistical analysis report published by the Committee of Brain Tumors Registry of Japan indicated that the incidence of choriocarcinoma was $3.2 \%$ (36 cases) out of 1127 primary intracranial GCT cases (5). Additionally, Matsutani et al analyzed 153 cases of histologically verified intracranial GCTs and revealed that the incidence of choriocarcinoma was 3.3\% (3). Due to this extremely low incidence, the majority of publications refer to case reports (6-11), and few studies have compared the treatment and prognosis $(1,3)$. Matsutani et al classified intracranial GCT into three therapeutic groups: those with good, intermediate and poor prognoses. PICCCs were classified into the poor prognosis group, and this group required more aggressive chemotherapy and radiotherapy (3). A recent review article presented an algorithm for diagnostic evaluation and treatment of pineal or suprasellar tumors (12). The author suggested that PICCCs require combinational therapy comprising chemotherapy, radiotherapy and resection. However, there is currently no clear national consensus for the optimal treatment of PICCCs.

Shinoda et al reviewed 66 PICCC/GCTs with high $\beta$-HCG levels and revealed that their median survival time and their one-

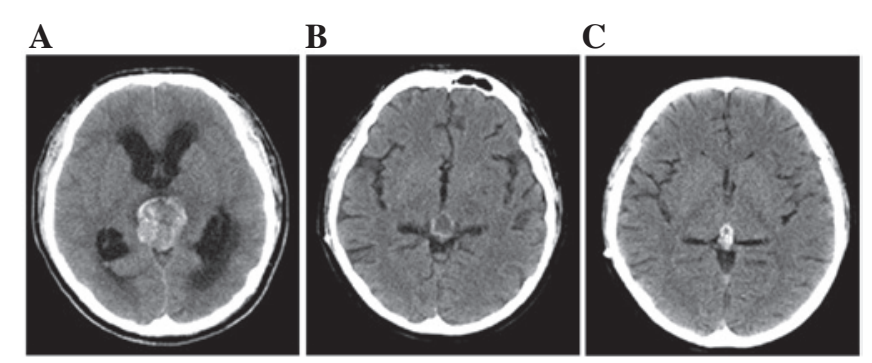

Figure 1. CT scans. (A) Initial imaging demonstrates acute hydrocephalus and a huge mass occupying the pineal region. (B) Following completion of the first course of synchronous chemotherapy and radiotherapy, imaging demonstrated an approximate $45 \%$ reduction in the tumor mass and disappearance of the hydrocephalus. (C) Following completion of the additional three courses of chemotherapy, the tumor mass was reduced by a further $63 \%$. CT, computed tomography.

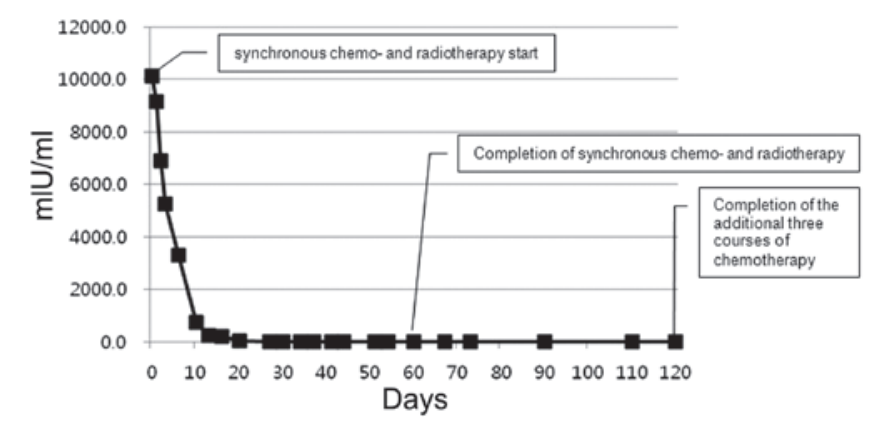

Figure 2. Serial serum $\beta$-HCG levels in the patient during treatment. Following completion of the first course of synchronous chemotherapy and radiotherapy, serum $\beta-\mathrm{HCG}$ was normalized at $0.2 \mathrm{mIU} / \mathrm{ml}$.

and two-year survival rates were 22 months, $61.2 \%$ and $49.8 \%$, respectively (1). Among these 66 patients, 34 (52\%) succumbed within one year of diagnosis and $14(21 \%)$ succumbed within one month of diagnosis. Early mortality is commonly associated with tumor hemorrhage. This suggests that the prognosis of these patients may be improved if the initial treatment focuses on the prevention of tumor hemorrhage.

To minimize early mortality arising from tumor hemorrhage, we planned the following treatment regimen. First, we did not conduct a tumor biopsy for pathological confirmation. $\beta$-HCG is regarded to be more characteristic of $\beta$-HCG-secreting tumors. Markedly elevated $\beta$-HCG levels are strongly suggestive of choriocarcinomas, while mild elevations can be observed in other GCTs of syncytiotrophoblastic giant cells (1). In a study by Matsutani et al, all patients with pure choriocarcinomas or mixed GCTs with a choriocarcinoma element had highly elevated serum $\beta$-HCG levels of $>2,000 \mathrm{IU} / 1$, while GCT patients without a choriocarcinoma element had serum $\beta$-HCG levels of $<770$ IU/l (3). The serum $\beta$-HCG level in our patient was $>1,0147 \mathrm{mIU} / \mathrm{ml}$. The patient had a tumor arising from the pineal region with hemorrhage, hydrocephalus and a history of progressive consciousness deterioration. A biopsy was not conducted due to the limited access and high hemorrhagic risk. We diagnosed the tumor as PICCC based only on extremely elevated $\beta$-HCG levels, and could prevent tumor hemorrhage by biopsy. Secondly, we selected synchronous chemotherapy and radiotherapy as the 
first-line treatment. Sakurada et al reported that tumor hemorrhage occurred when radiotherapy was conducted as an initial treatment (13). Shinoda et al stated that initial biopsy and radiotherapy may lead to tumor hemorrhage in PICCCs (1). Thus, we determined that it was dangerous to start treatment with radiation alone. Kageji et al reported a case of successful neoadjuvant synchronous chemotherapy and radiotherapy for disseminated PICCC (8). The patient underwent induction chemotherapy in parallel with radiotherapy as the initial treatment. Following radiotherapy and the second course of chemotherapy, the patient underwent radical tumor surgery. There was no evidence of hemorrhage during neoadjuvant treatment. Based on this, we immediately started initial treatment comprising synchronous chemotherapy and radiotherapy. The patient did not undergo fatal events by hemorrhage during synchronous therapy, and once the first course of synchronous chemotherapy and radiotherapy was complete, the previously observed hemorrhage had disappeared. Thirdly, we did not attempt to remove the remaining mass. The patient had a tumor arising from the pineal region, and due to the number of neurovascular structures in this area, it is a surgically inaccessible area of the brain (14). Despite the fact that the tumor hemorrhage had disappeared following the first course of treatment, the hemorrhagic risk of PICCC remained. Chan et al reported a case of successful treatment with chemotherapy and radiotherapy without surgery (15). We planned three consecutive courses of chemotherapy without surgery. The tumor mass was reduced by $63 \%(14 \times 12 \mathrm{~mm})$, and the patient's abnormal neurological symptoms were fully recovered.

To date, the majority of the literature suggests that intensive chemotherapy, radiation and tumor resection offer the best chance of treating PICCCs. We agree with this opinion; however, we suggest that a personal approach to prevent early tumor hemorrhage is the most important factor.

Although PICCCs are rare, their propensity for fatal hemorrhage and extraneural/CSF metastasis results in a poor prognosis. Our patient exhibited severe consciousness disturbances and a high level of serum $\beta$-HCG. Since the initial imaging study demonstrated a tumor mass with hemorrhage in the pineal region, we immediately began synchronous chemotherapy and radiotherapy. Subsequently, our patient underwent three courses of chemotherapy, each comprising the same ICE regimen. The residual tumor mass was not resected due to limited access and hemorrhagic risk. Nearly two years following the initial diagnosis, the patient is alive without tumor progression and with normal neurological functions.

In conclusion, we reported a rare case of PICCC. We diagnosed our patient with the aid of serum $\beta$-HCG level without biopsy, and immediately started induction therapy comprising synchronous chemotherapy and radiotherapy. Without surgery, we delivered ICE chemotherapy as consolidation treatment. These treatments led to tumor regression and marked improvements in the patient's signs and symptoms. Nearly two years post-diagnosis, follow-up imaging has demonstrated no tumor progression. This is the first report of successful synchronous chemotherapy and radiotherapy, followed by three courses of consecutive chemotherapy, to treat a patient diagnosed with PICCC by focusing the initial treatment on the prevention of tumor hemorrhage.

\section{References}

1. Shinoda J, Sakai N, Yano H, Hattori T, Ohkuma A and Sakaguchi H: Prognostic factors and therapeutic problems of primary intracranial choriocarcinoma/germ-cell tumors with high levels of HCG. J Neurooncol 66: 225-240, 2004.

2. Jennings MT, Gelman R and Hochberg F: Intracranial germ-cell tumors: natural history and pathogenesis. J Neurosurg 63: 155-167, 1985.

3. Matsutani M, Sano K, Takakura K, et al: Primary intracranial germ cell tumors: a clinical analysis of 153 histologically verified cases. J Neurosurg 86: 446-455, 1997.

4. Matsutani M: Clinical management of primary central nervous system germ cell tumors. Semin Oncol 31: 676-683, 2004.

5. The Committee of the Brain Tumor Registry of Japan: Report of Brain Tumor Registry of Japan (1969-1993). 10th edition. Neurol Med Chir 40 Suppl: 1-106, 2000.

6. Kawakami Y, Yamada O, Tabuchi K, Ohmoto T and Nishimoto A: Primary intracranial choriocarcinoma. J Neurosurg 53: 369-374, 1980.

7. Fujii T, Itakura T, Hayashi S, Komai N, Nakamine H and Saito K: Primary pineal choriocarcinoma with hemorrhage monitored by computerized tomography. Case report. J Neurosurg 55: 484-487, 1981.

8. Kageji T, Nagahiro S, Matsuzaki K, et al: Successful neoadjuvant synchronous chemo- and radiotherapy for disseminated primary intracranial choriocarcinoma: case report. J Neurooncol 83: 199-204, 2007.

9. Nitta H, Yamashita J, Nomura M and Igarashi N: Cervical spinal cord infarction after surgery for a pineal region choriocarcinoma in the sitting position: case report. Neurosurgery 40: 1082-1085; discussion 1085-1086, 1997.

10. Taomoto K, Tamaki N, Obora Y, Nakai K and Sato H: Intracranial human chorionic gonadotropin producing tumors (author's transl). Neurol Med Chir (Tokyo) 16: 411-418, 1976 (In Japanese).

11. Marx M, Beck JD, Grabenbauer GG, Fahlbusch R and Dörr HG: Gonadotrophin-independent puberty in a boy with a beta-HCG-secreting brain tumour. Horm Res 54: 44-48, 2000.

12. Kyritsis AP: Management of primary intracranial germ cell tumors. J Neurooncol 96: 143-149, 2010

13. Sakurada K, Kayama T, Kawakami K, Saino M and Sato S: A successfully operated case of choriocarcinoma with recurrent intratumoral hemorrhage. No Shinkei Geka 28: 67-72, 2000 (In Japanese).

14. Yamamoto I: Pineal region tumor: surgical anatomy and approach. J Neurooncol 54: 263-275, 2001.

15. Chan HS, Humphreys RP, Hendrick EB, Chuang SH, Fitz CR and Becker LE: Primary intracranial choriocarcinoma: a report of two cases and a review of the literature. Neurosurgery 15: $540-545,1984$. 